\title{
Concentration of Astragalus Polysaccharide by Polysulfone- Activated Carbon Modified with Nitric Acid Blend Ultrafiltration Membranes
}

\author{
Hao Dan-Dan, Chen Jian-Qiu, Liao Xin-Qiao, Zheng Yan and Ji Yi-Bing \\ Department of Analytical Chemistry \& Department of Environmental Science, China Pharmaceutical \\ University, Nanjing 210009, China
}

\begin{abstract}
In this study, Astragalus polysaccharides were concentrated by ultrafiltation membranes prepared by blending polysulfone (PSF) with activated carbon modified with nitric acid(HAC) by immersion precipitation phase inversion method. Water flux and retention to bovine serum albumin of the membranes were investigated. The morphology of membranes were also observed by scanning electron microscopy (SEM). In order to obtain best concentration performance, effects of operating pressure, feeding temperature, operating time, feeding concentration and $\mathrm{pH}$ value on concentration and extraction of astragalus polysaccharide were studied. The best conditions for concentration were as follows: operating pressure is $0.4 \mathrm{MPa}$, feeding temperature is $25^{\circ} \mathrm{C}$, feeding concentration is $1 \mathrm{~g} \cdot \mathrm{L}^{-1}, \mathrm{pH}$ value is neutral. Under these conditions, the ultrafiltration membrane flux was $62.6 \mathrm{~L} \cdot \mathrm{m}^{-2} \cdot \mathrm{h}^{-1}$, the retention to astragalus polysaccharide was $91.2 \%$, and the attenuation rate of water flux was $12.5 \%$ in one hour.
\end{abstract}

Keywords: Polysulfone, modified activated carbon, Immersion precipitation phase inversion method, ultrafiltration, Astraglus polysaccharide.

\section{INTRODUCTION}

Astragalus, commonly known as one of Chinese herbal medicines, is the dried roots of Astragalus membranaceus (Fisch) Bge. Var. mongholicus (Bge) Hsiao or Astragalus membranaceus (Fisch) Bge. The Radix Astragali is one of the most popular healthpromoting herbal medicines commonly used in China [1], its use is traced back to more than 2000 years, and were recorded in Shen Nong's Materia Medica written in the Han dynasty. Astragalus polysaccharide (APS) is one of the main active ingredients in Astragalus. A series of studies have intensively focused on the physiological functions of APS due to its enhanced immunity [2], hypoglycemic, antitumor, antiviral, antiradiation, anti-aging and other pharmacological effects [3]. Apart from studying pharmacological activity of APS, extraction methods [4] are another hot spot for researchers. Traditional polysaccharide extraction methods, such as water extraction and alcohol precipitation, have problems of reagent consumption, low efficiency, process complication and incomplete impurities removal. Membrane technology, having a good prospect of application, has high advantages of simple operation, energy savings, environmental protection, no phase change, and no biological activity loss. Membrane based separation technologies have found a wide spread use in biotechnological,

*Address correspondence to this author at the Department of Analytical Chemistry \& Department of Environmental Science, China Pharmaceutical University, Nanjing 210009, China; Tel: +86 (25) 8618 5190; Fax: +86 (25) 8618 5190; E-mail: jiyibing_cpu@163.com pharmaceutical and food industries or to treat other industrial effluents. In the case of Ultrafiltration, UF membranes have been widely applied in separation technologies such as virus removal, low concentration effluents treatment or water purification [5]. Molecular weight of APS(more than $10 \mathrm{kDa}$ ) is a distinctive difference compared with saponins. Therefore, it is possible to use ultrafiltration membranes for better isolating Astragalus polysaccharides and saponins, which can conquer shortcomings of the traditional water extraction and alcohol precipitation that can not separate polysaccharides from flavonoids and saponins [6].

As far as we know, there are few reports about extracting APS through ultrafiltation membrane [7]. In the present investigation, blend membranes based on PSF/HAC were prepared and used for extracting and concentrating Astragalus polysaccharides. It can be seen that the PSF/HAC blend membranes show good antifouling performance in the ultrafiltration process. The effect of operating pressure, feeding temperature, feeding concentration, feeding $\mathrm{pH}$ value and operating time of the solid to liquid ultrafiltration behavior were discussed, providing a theoretical and experimental reference for the industrial production applications.

\section{EXPERIMENTAL}

\subsection{Materials and Instruments}

Astragalus polysaccharides ( $\mathrm{Mw} \geq 10 \mathrm{kDa}$ ) was provided by Fujian benefit Biotechnology Co., Ltd. 
Polysulfone (PSF) was purchased from Shanghai Shu Austrian Chemical Industry Co., Ltd. Dimethyl acetamide (DMAc System) and analytical reagent grade sulfuric acid were obtained from Nanjing Chemical Reagent Co., Ltd. Chemically pure grade polyethylene glycol 400 (PEG-400) and analytical reagent grade glucose were bought from Sinopharm chemical Reagent Co., Ltd. Activated carbon (200 mesh) was purchased from Jiangsu Zhuxi Activated Carbon Co., Ltd. Analytical reagent grade phenol was bought from Shanghai Ling Feng chemical Reagent Company. Analytical reagent grade nitric acid was purchased from pilot Chemical Corporation. GZX-9070 thousand MBE, the number of significant blast oven from Shanghai Xun Industrial Co., Ltd. medical Equipment Factory; Hitachi S3400 scanning electron microscope(Japan Hitachi); BS124S electronic analytical balance, raceDoric scientific Instruments (Beijing) Co., Ltd.; HH-2 digital display thermostat water bath, Guohua Electric Appliance Co., Ltd.; JH752 UV-Vis spectrophotometer, Shanghai Jinghua Science and Technology instrument Co., Ltd.; membrane performance tester, Hangzhou moon Springs machinery and Equipment Co.company were used.

\subsection{Preparation of the Oxidative Modification Activated Carbon}

The carbon was firstly washed to remove impurities on the surface of activated carbon. $5 \mathrm{~g}$ activated charcoal was dissolved in $25 \mathrm{~mL}$ of $20 \%$ nitric acid solution under constant mechanical oscillation with a totational speed of $200 \mathrm{r} / \mathrm{min}$ at constant temperature $30{ }^{\circ} \mathrm{C}$ for $6 \mathrm{~h}$. Then the activated carbon was washed by deionized water until neutral, and was dried at 105 ${ }^{\circ} \mathrm{C}$ for $12 \mathrm{~h}$. The modified activated carbon was recorded as HAC-20.

\subsection{Preparation of Composite Membranes}

The membrane was prepared by immersion precipitation phase inversion method in DMAc as the solvent, containing $15 \mathrm{wt} \%$ PSf, 8\% PEG400 and 1 wt\% active carbon relative to the PSf mass fraction. The casting solution was then heated at $50{ }^{\circ} \mathrm{C}$ for $1 \mathrm{~h}$ until the whole solution got fully swelled, and escalated heating temperature to $70{ }^{\circ} \mathrm{C}$ with a fully stirring for $6 \mathrm{~h}$ until a transparent homogeneous casting solution formed with $24 \mathrm{~h}$ deaeration followed.

A certain thickness of the membrane was evenly scraped by a homemade scratch on a piece of glass plate and can be controlled through the copper wire diameter evenly curled on scraping rods. The glass plate was kept in ambient temperature and humidity during membrane casting as specified. Solvent was allowed to evaporate for 60 s and then were immersed in pure water gelation bath. The off-membranes were soaked in water for $24 \mathrm{~h}$ with freshing water altered every 5 hours to remove the residual solvents and additives, and dried at $60^{\circ} \mathrm{C}$ for use.

\subsection{Characterization of Membrane}

\subsubsection{Permeation Flux (J) and Rejection (R)}

The membrane flux is a measure of permeability for a membrane. Through UF cross flow filtration experimental set-up, the membrane flux of the pure water was determined under room temperature and 0.2 MPa operating pressure for $0.5 \mathrm{~h}$ with the $0.5 \mathrm{~h}$ preload. The permeation flux was defined as formula (1):

$\mathrm{J}=\mathrm{V} / \mathrm{At}$

$\mathrm{J}$ stands for membrane flux of the feed solution, $\mathrm{L} \cdot$ $\mathrm{m}^{-2} \mathrm{~h}^{-1} ; \mathrm{V}$ is the volume of the liquid permeated $(\mathrm{L}) ; A$ is the membrane effective area $\left(\mathrm{m}^{2}\right) ; t$ is the required permeated time(h)

Rejection was characterized with $1 \mathrm{~g} / \mathrm{L}$ BSA aqueous solution after the membrane was previously filtered with pure water until flux was steady. The concentrations of BSA in permeate and feed were determined by an UV-spectrophotometer (JH752, China) at $280 \mathrm{~nm}$. It was calculated according to formula (2):

$R=1-C_{p} / C_{f}$

where $C_{p}$ and $C_{f}$ are the concentrations of protein in permeate and initial feed, respectively, $\mathrm{g} / \mathrm{L}$.

\subsubsection{Porosity $(P)$}

Membrane porosity was measured in the method of dry-wet weight. The membrane immersed in distilled water was weighed after mopping superficial water with filter paper. Then the wet membrane was puted in an air-circulating oven at $60{ }^{\circ} \mathrm{C}$ for $24 \mathrm{~h}$. At last, the membrane was further dried in a vacuum oven at $80^{\circ} \mathrm{C}$ for $24 \mathrm{~h}$ before measuring the dry mass. On the basis of the two masses (the wet sample mass and the dry sample mass), the porosity of membrane was calculated using formula (3) [8]:

$P(\%)=100\left(m_{w}-m_{d}\right) /\left(A^{*} \delta^{\star} \rho_{w}\right)$

where $P$ is the porosity of membrane, $m_{\mathrm{w}}$ is the wet sample mass $(\mathrm{g}), m_{\mathrm{d}}$ is the dry sample mass $(\mathrm{g}), \rho_{w}$ is 
the density of pure water $\left(\mathrm{g} / \mathrm{cm}^{3}\right), A$ is the area of membrane in wet state $\left(\mathrm{cm}^{2}\right)$ and $\delta$ is the thickness of membrane in wet state $(\mathrm{cm})$. In order to minimize experimental error, each membrane was measured for three times and calculated average.

\subsubsection{Morphological Studies}

The dry membranes were cut into pieces of desired sizes and immersed in liquid nitrogen for seconds to fracture the membranes. The membranes surface were gold-sputtered to provide electrical conductivity. The surface morphology and cross-sectional structure of the membrane were observed by Hitachi $\mathbf{S} 3400$ scanning electron microscope (SEM).

\subsection{Determination of Astragalus polysaccharide}

The Astragalus polysaccharide concentrations were measured by a UV/Vis spectrophotometer (UV 752, China) at $490 \mathrm{~nm}$ [9]. Through fitting calculation, the linear regression equation is $y=16.175 x+0.0431, r=$ 0.9975 , and the concentration and absorbance show a good linear relationship within the range of 0.005 $0.080 \mathrm{~g} / \mathrm{L}$.

\subsection{Determination of Polysaccharide Retention Rate}

Under certain Astragalus sugar concentration, temperature and operating pressure, the polysaccharide retention rate was determined through the membrane test device (Figure 1) by collecting permeated solution and by measuring the absorbance of feed solution and permeated solution at $490 \mathrm{~nm}$. All experiments were carried out at room temperature. The volume of the test device is $6 \mathrm{~L}$. The concentration of feed solution and permeated solution were calculated according to the standard curve equation. The retention rate of APS were measured in accordance with the following formula:

$\mathrm{R}=\left(\mathrm{C}_{0}-\mathrm{C}_{1}\right) / \mathrm{C}_{0}$

Where: $\mathrm{R}$ is the retention rate; $\mathrm{C}_{0}$ and $\mathrm{C}_{1}$ is the concentration of feed solution and permeated solution respectively $\left(g \cdot L^{-1}\right)$.

\section{RESULTS AND DISCUSSION}

\subsection{Characterization of Membrane}

\subsubsection{Permeation Flux (J) and Rejection (R)}

Compared with PSF membrane and PSF / AC membrane, PSF / HAC (HAC-20) composite films

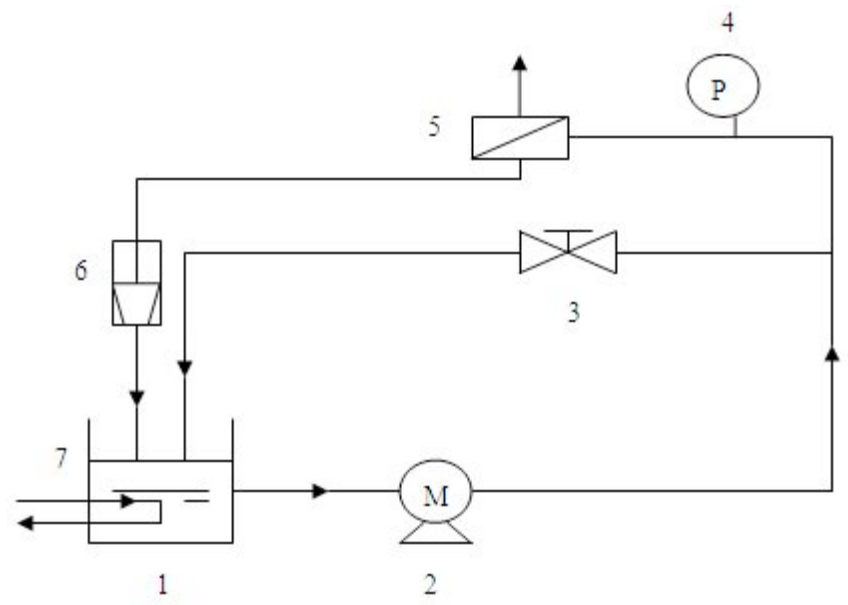

Figure 1: Schematic diagram of the membrane performance test system.

1 Feed tank 2 High-pressure pump 3 Regulating valve 4 Pressure gauge 5 Carrier film sets 6 Rotameter 7 Temperature control system.

shows the best performance (Table 1), due to the oxide modified activated carbon within HAC-20 contains larger hydrophilic group. According to the principle that similarity can be dissolved easily to each other, the permeated water through the membrane suffers less resistance which is conducive to water infiltration, and induces a larger water flux [10]. Meanwhile, adsorption synergistic effect of activated carbon can ensure the membrane of higher rejection rate. Performance of modified activated carbon composite membranes has improved significantly compared with the unmodified activated carbon membrane.

Table 1: Performance of the Membrane Filled with Modified Activated Carbon

\begin{tabular}{|c|c|c|c|}
\hline Type of membrane & PSF & PSF/AC & PSF/HAC \\
\hline \hline $\mathrm{J} / \mathrm{L} \cdot \mathrm{m}^{-2} \cdot \mathrm{h}^{-1}$ & 102.8 & 53.3 & 116.3 \\
\hline $\mathrm{R} / \%$ & 83.4 & 87.5 & 90.4 \\
\hline
\end{tabular}

\subsubsection{Porosity (P)}

As for oxidative modification of activated carbon composite membranes, surface porosity is significantly improved to $72.8 \%$ with more uniform distribution, which can be helpful to form a sharp split.

\subsubsection{Morphological Study}

Figure 2 shows the microstructure of the composite membranes, consisting of top surface and crosssections. Figure $\mathbf{2 a}$, $\mathbf{b}$ show the top surface and crosssectional of PSF membranes, while Figure 2c, d are the top surface and cross-sectional of PSF/HAc 


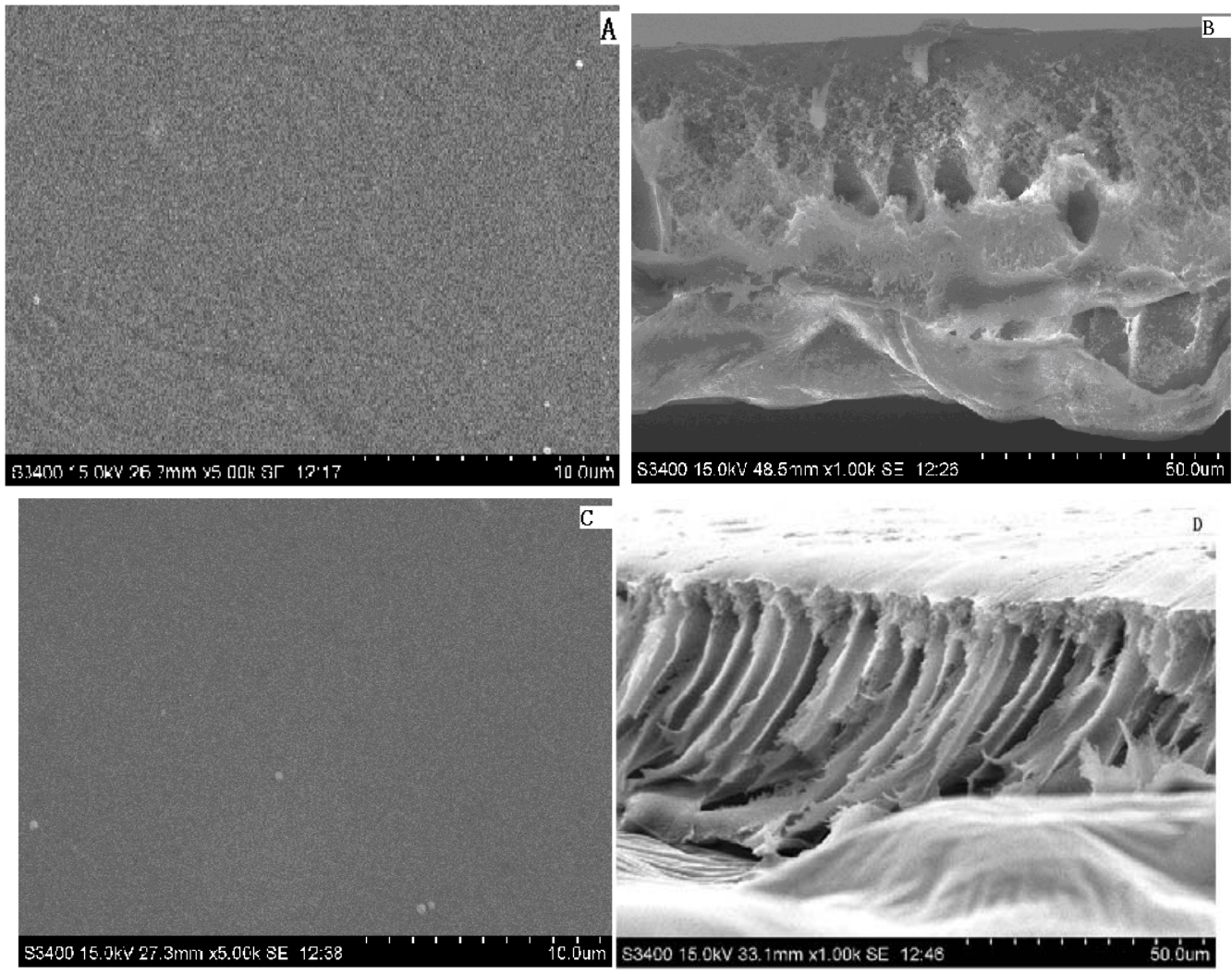

Figure 2: SEM micrographs of surface and cross-section of PSF and PSF/HAC membranes.

(A) Surface of PSF membrane (B) Cross-section of PSF membrane (C) Surface of PSF/HAC membrane (D) Cross-section of PSF/HAC membrane.

membranes. These two kinds of membrane above are constructed by surface dense layer and the internal porous support layer. From It is observed from the figures that in the absence of activated carbon, the pores are very small with the cross section full of cavernous and finger-shaped hole transition structure having larger aperture.

This is because, on one hand, the activated carbon is distributed in the membrane surface and pore walls; on the other hand, the cross-sectional structure of the composite membrane finger-like, which has good underlying connectivity with good aperture. This sort of morphology and internal structure is conducive to higher retention rate [11-12] with appropriate water flux guaranteed, which are consistent with the separation performance of composite membranes.

\subsection{The Effect of Operating Pressure on the Ultrafiltration Performance}

Pressure is the motivation for ultrafiltration separation, which somehow directly determines separation performance of the membrane. Under the condition of Astragalus polysaccharide concentration $0.5 \mathrm{~g} \cdot \mathrm{L}^{-1}$ with feed temperature at $25^{\circ} \mathrm{C}$, the effect of operating pressure on the ultrafiltration process of sample solution was observed, which is shown in Figure 3.

Under the relative small operating pressure, the increases of pressure can help material fluid better permeate through the membrane, leading membrane flux significantly increased. However, after reaching a certain level, the pressure increases would make the film appear the concentration polarization phenomenon. A surface gel layer was gradually formed on the membrane, resulting in increased resistance and the increasing trend of the membrane flux slow down.

The retention rate is reduced with a small margin when pressure increased. This may due to pressure increase would accelerate the polysaccharide molecules permeation, which leads some of the polysaccharide molecules not retained [13]. In addition, the gel layer formation on the membrane surface would induce solid to liquid adsorption, which decreases the transmittance of the membrane. Both two factors affect the transmittance of the polysaccharide, so with the increase of the pressure the change of rentention rate is relatively flat. Considering the efficiency of 


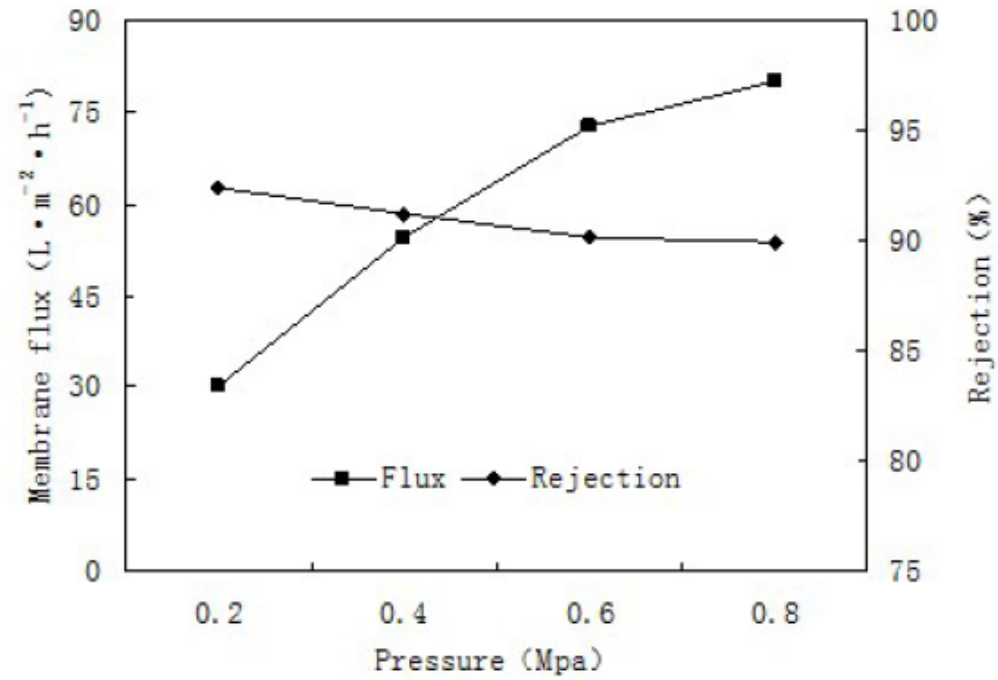

Figure 3: Effect of operating pressure on ultrafiltration.

The tested membranes: the PSF/HAC blend membranes; The operating condition: the feed concentration 0.5 $\mathrm{g} \cdot \mathrm{L}^{-1}$ and feed temperature at $25^{\circ} \mathrm{C}$ in room temperature.

concentrating polysaccharides and energy costs, we adopted $0.4 \mathrm{MPa}$ as the operating pressure for ultrafitration process.

\subsection{The Effect of Feed Temperature on the Ultrafiltration}

On the premise of operating pressure $0.4 \mathrm{MPa}$ and feed concentration $0.5 \mathrm{~g} \cdot \mathrm{L}^{-1}$, the effect of feed temperature for ultrafiltration of Astragalus sugar solution was investigated (Figure 4).

The figure shows that the membrane flux increases with higher temperatures, which may out of feeding liquid viscosity decrease in a certain temperature range that can magnify diffusion coefficient, mass transfer coefficient [14] and polysaccharide solubility. Moreover, the affinity between the solute and water is intensified with concentrated polarization reduced, so the permeate flux increases. On the contrary, retention rate decreases as the temperature rises, because the higher temperature causes membrane swelling, making the membrane pore to increase so as to resulting in the change of the pore size distribution. Changes in physical and chemical properties of the membrane may occur if the temperature is too high [15], resulting in reduced membrane rejection rate. Take the separation and stability of the feeding liquid into consideration, we

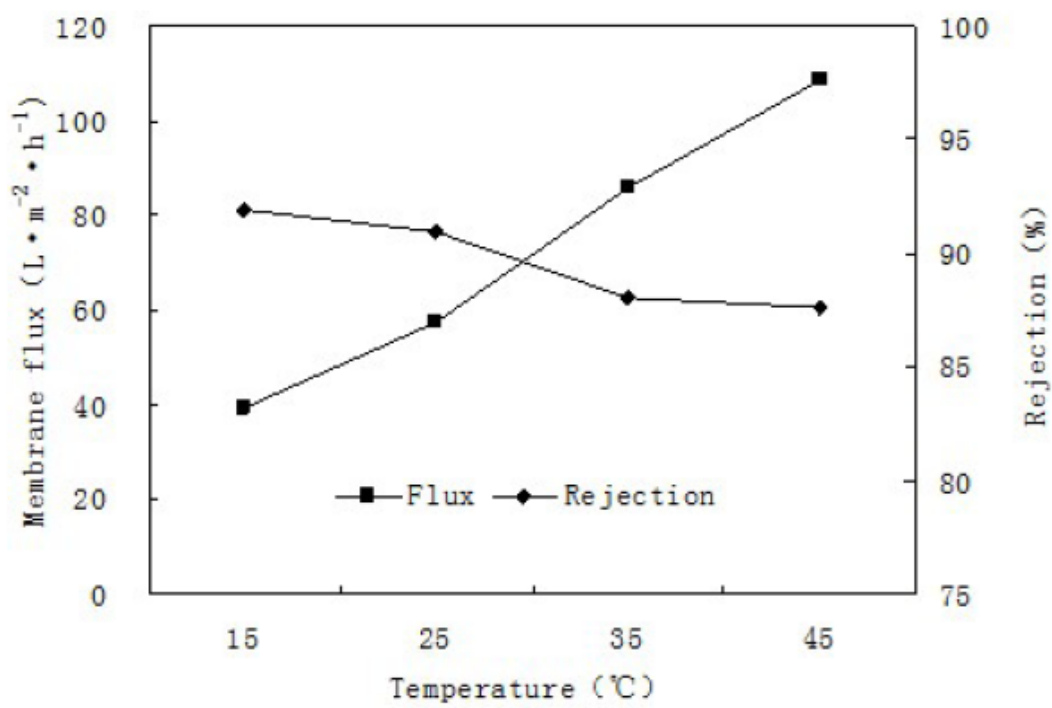

Figure 4: Effect of feeding temperature on ultrafiltration.

The tested membranes: the PSF/HAC blend membranes; The operating condition: perating pressure $0.4 \mathrm{MPa}$ and feed concentration $0.5 \mathrm{~g} \cdot \mathrm{L}^{-1}$ in room temperature. 


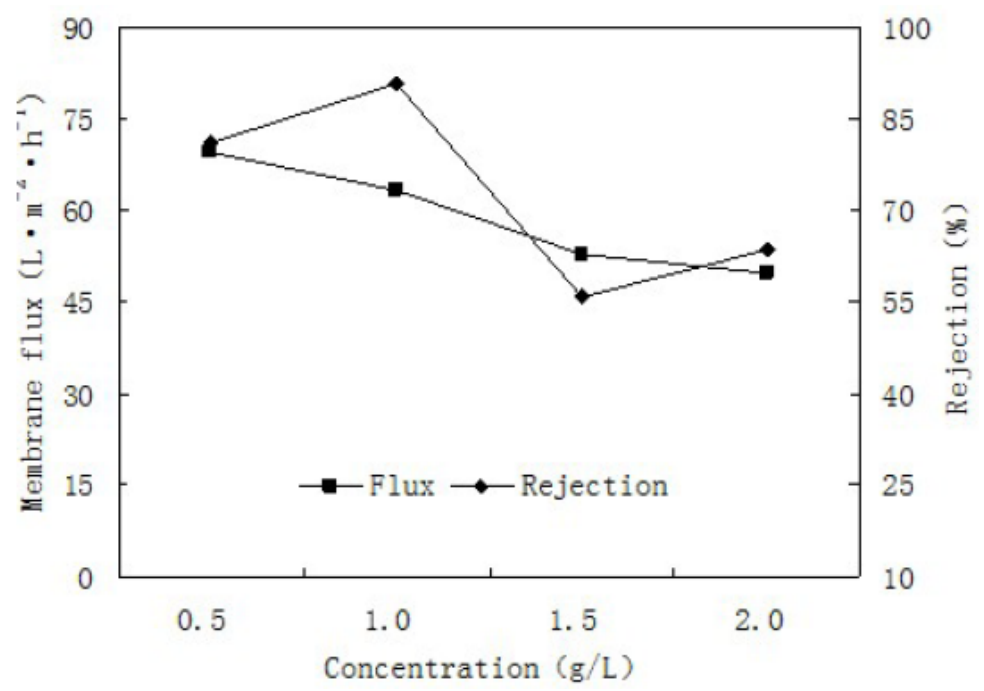

Figure 5: Effect of feeding concentration on ultrafiltration.

The tested membranes: the PSF/HAC blend membranes; The operating condition: operating pressure 0.4 MPa and feed temperature at $25^{\circ} \mathrm{C}$ in room temperature.

select the room temperature $25^{\circ} \mathrm{C}$ as appropriate ultrafiltration temperature.

\subsection{The Effect of Feed Concentration on the Ultrafiltration}

At an operating pressure of $0.4 \mathrm{MPa}$ and temperature conditions of $25{ }^{\circ} \mathrm{C}$, the effect of feed concentration of Astragalus polysaccharide is shown in Figure 5.

With the increase of polysaccharide concentration in the feed solution, the membrane flux decline. This is because the material viscosity increases as the feed concentration increases, which can create the membrane concentration polarization phenomenon [16]. At the same time, excessive concentration of solute would deposit on the membrane surface which the film hole would be plugged to a certain extent, enhacing the solid to liquid resistance, and thus a decreasing membrane flux is observed. Because of excessive concentration of polysaccharide, the chance of polysaccharide transmittance is higher, coupled with the continuing decline of membrane flux, the retention rate firstly gets small amplitude and then has an overall downward trend. It can be concluded that the membrane flux and rejection rate would have a better

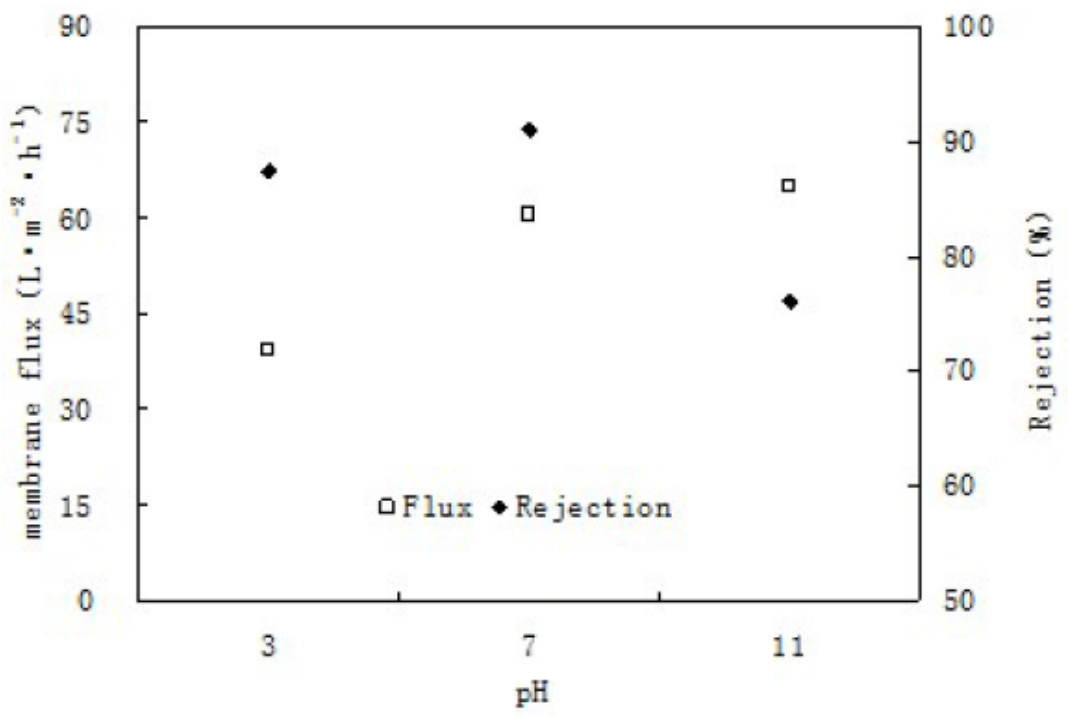

Figure 6: Effect of feeding pH on ultrafiltration.

The tested membranes: the PSF/HAC blend membranes; The operating condition: operating pressure $0.4 \mathrm{MPa}$, feed concentration $1.0 \mathrm{~g} / \mathrm{L}^{-1}$ and feed temperature at $25^{\circ} \mathrm{C}$ in room temperature. 
function when the feed concentration smaller, but too low of the feed concentration will result in high operating costs. Therefore, $1.0 \mathrm{~g} \cdot \mathrm{L}^{-1}$ of Astragalus polysaccharide as the beginning of the feed concentration for ultrafiltation is selected.

\subsection{The Effect of the Feed Solution $\mathrm{pH}$ on the Ultrafiltration}

Under the condition of Operating pressure $0.4 \mathrm{MPa}$ with feed concentration of $1.0 \mathrm{~g} \cdot \mathrm{L}^{-1}$ and ultrafiltration temperature at $25{ }^{\circ} \mathrm{C}$ the effect of feed solution $\mathrm{pH}$ on the ultrafiltration process was observed, which is shown in Figure 6.

The figure shows that membrane flux is increasing with the increase of $\mathrm{pH}$ and has the best performance under alkaline conditions, while polysaccharide retention rate is the best in neutral conditions. The reason may be different solubility of Astragalus polysaccharide at different $\mathrm{pH}$ values and the dissociation of oxygen-containing acidic groups in the membrane under basic conditions, which increases the hydrophilicity of the membrane. On the other hand, isoelectric point $(\mathrm{pl})$ of the most vegetable proteins are below 7 [17], and in acidic conditions, especially at the isoelectric point, precipitation of a small amount of protein impurities would aggregate formation of membrane surface gel layer [18], which can affect the membrane flux and rejection rate. Therefore, nearneutral $\mathrm{pH}$ value conditions are selected for the material.

\subsection{The Effect of Operating Time on the Ultrafiltration}

Under the condition of Operating pressure $0.4 \mathrm{MPa}$ with feed concentration of $1.0 \mathrm{~g} \cdot \mathrm{L}^{-1}$ and ultrafiltration temperature at $25^{\circ} \mathrm{C}$ the impact of operating time on the ultrafiltration process and the antifouling property of ultrafiltration membrane were observed, which is shown in Figure 7.

It shows that the membrane flux in the ultrafiltration process declines with the extension of the film running time and the retention rate has a smaller change compared with membrane flux, which also has a slower speed after running $40 \mathrm{~min}$ and finally stabilized. This is consistent with the attenuation law of the membrane flux [19]. The initial permeate flux was $69.4 \mathrm{~L} \cdot \mathrm{m}^{-2} \cdot \mathrm{h}^{-1}$ with $60.4 \mathrm{~L} \cdot \mathrm{m}^{-2} \cdot \mathrm{h}^{-1}$ after running $60 \mathrm{~min}$, and the overall decline is $13.0 \%$, which indicates that the membranes have good antifouling performance.

\section{CONCLUSIONS}

The separation performance of PSf/HAC composite membrane and its application to concentration of APS process were studied. Compared with PSf membrane and PSF/AC membrane, PSF/HAC (HAC-20) composite films shows the best performance with water flux $116.3 \mathrm{~L} \cdot \mathrm{m}^{-2} \cdot \mathrm{h}^{-1}$, the BSA retention rate of $90.4 \%$ and $72.8 \%$ porosity, due to the oxide modified activated carbon within HAC-20 contains larger hydrophilic group. In addition, adsorption synergistic effect of

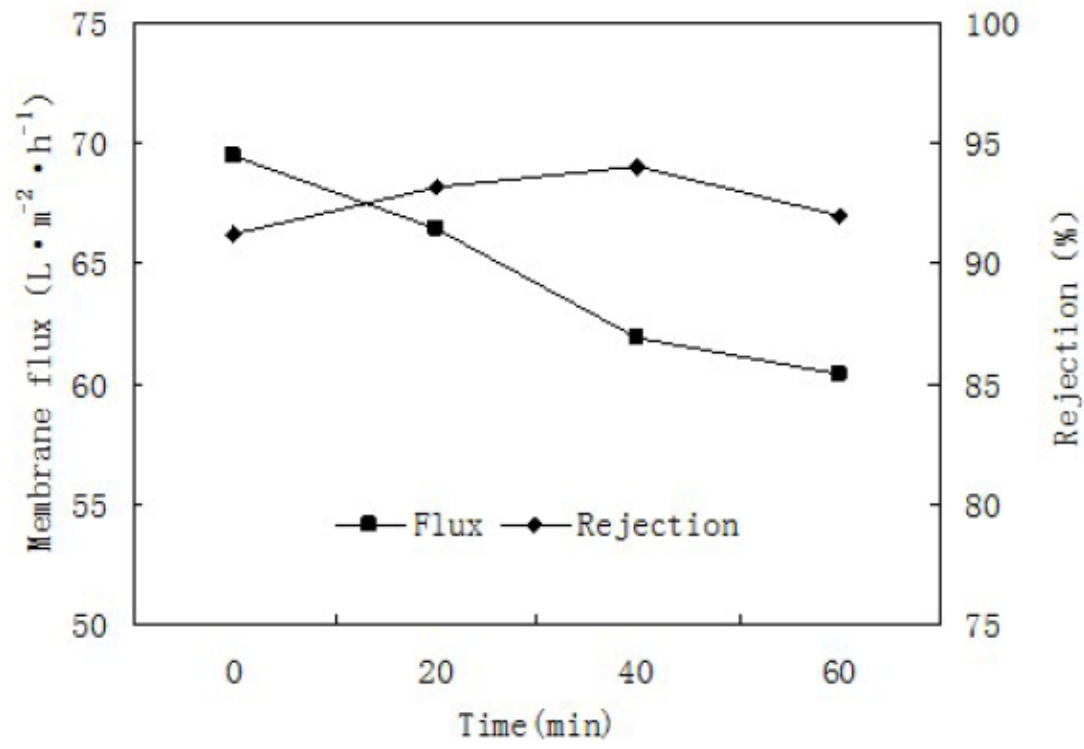

Figure 7: Effect of operating time on ultrafiltration.

The tested membranes: the PSF/HAC blend membranes; The operating condition: operating pressure $0.4 \mathrm{MPa}$, feed concentration $1.0 \mathrm{~g} / \mathrm{L}^{-1}$ and feed temperature at $25^{\circ} \mathrm{C}$ in room temperature. 
activated carbon can ensure the membrane of higher rejection rate.

In order to acquire best concentration performance, effects of operating pressure, feeding temperature, feeding concentration, feeding $\mathrm{pH}$ value and operating time on concentration and extraction of astragalus polysaccharide were studied. The best conditions for concentration were as follows: operating pressure is $0.4 \mathrm{MPa}$, feeding temperature is $25^{\circ} \mathrm{C}$, feeding concentration is $1 \mathrm{~g} \cdot \mathrm{L}^{-1}, \mathrm{pH}$ value is near neutral. Under these conditions, the flux was $62.6 \mathrm{~L} \cdot \mathrm{m}^{-2} \cdot \mathrm{h}^{-1}$, the retention to astragalus polysaccharide was $91.2 \%$, and the attenuation rate of water flux was $12.5 \%$ in one hour. These results provide a theoretical and experimental reference for the industrial production applications.

\section{REFERENCES}

[1] Yan H, Xie Y, Sun S, et al. Chemical analysis of Astragalus mongholicus polysaccharides and antioxidant activity of the polysaccharides[J]. Carbohydr Polym 2010; 82: 636-40. Available from: http://www.sciencedirect.com/science/article/ pii/S0144861710004248

http://dx.doi.org/10.1016/j.carbpol.2010.05.026

[2] Qiu H, Cheng G, Xu J, et al. Effects of Astragalus Polysaccharides on Associated Immune Cells and Cytokines in Immunosuppressive Dogs[J]. Proc Vaccinol 2010; 2: 2633. Available from: http://www.sciencedirect.com/science/ article/pii/S1877282X1000007X

http://dx.doi.org/10.1016/j.provac.2010.03.006

[3] Kong X, Hu Y, Rui R, Wang D, Li X. Effects of Chinese herbal medicinal ingredients on peripheral lymphocyte proliferation and serum antibody titer after vaccination in chicken[J]. Int Immunopharmacol 2004; 4: 975-82. Available from: http://www.sciencedirect.com/science/article/pii/ S1567576904001031

\section{http://dx.doi.org/10.1016/j.intimp.2004.03.008}

[4] Yin J, Jiang Z, Yu H, et al. A new application of an aqueous diphase solvent system in one-step preparation of polysaccharide from the crude water extract of Radix Astragali by high-speed counter-current chromatography[J]. J Chromatogr A 2012; 1262: 92-97. Available from: http://www.sciencedirect.com/science/article/pii/S002196731 2013507

http://dx.doi.org/10.1016/j.chroma.2012.08.099

[5] Peinador RI, Calvo JI, Pradanos P, Palacio L, Hernandez A. Characterisation of polymeric UF membranes by liquid-liquid displacement porosimetry[J]. J Membr Sci 2010; 348: 23844. Available from: http://www.sciencedirect.com/science/ article/pii/S0376738809008072 http://dx.doi.org/10.1016/j.memsci.2009.11.008

[6] Mbareck C, Nguyen QT, Alaoui OT, Barillier D. Elaboration, characterization and application of polysulfone and polyacrylic acid blends as ultrafiltration membranes for removal of some heavy metals from water[J]. J Hazardous Mater 2009; 171: 93-101. Available from: http://www. sciencedirect.com/science/article/pii/S0304389409008796 http://dx.doi.org/10.1016/j.jhazmat.2009.05.123

[7] Xiao HB, Krucker M, Putzbach K, Albert K. Capillary liquid chromatography-microcoil ${ }^{1} \mathrm{H}$ nuclear magnetic resonance spectroscopy and liquid chromatography-ion trap mass spectrometry for on-line structure elucidation of isoflavones in
Radix astragali[J]. J Chromatogr A 2005; 1067: 135-43. Available from: http://www.sciencedirect.com/science/article/ pii/S0021967305000622 http://dx.doi.org/10.1016/i.chroma.2005.01.015

[8] Ma Y, Shi F, Zhao W, et al. Preparation and characterization of PSF/clay nanocomposite membranes with $\mathrm{LiCl}$ as a pore forming additive[J]. J Desalinat 2012; 303: 39-47. Available from: http://www.sciencedirect.com/science/article/pii/ S0011916412003876

http://dx.doi.org/10.1016/j.desal.2012.07.016

[9] Masuko T, Minami A, Iwasaki N, Majima T, Nishimura S-I, Lee YC. Carbohydrate analysis by a phenol-sulfuric acid method in microplate format[J]. Analyt Biochem 2005; 339: 69-72. Available from: http://www.sciencedirect.com/science/ article/pii/S0003269704009522

http://dx.doi.org/10.1016/j.ab.2004.12.001

[10] Lim JW, Lee J-M, Yun S-M, Park B-J, Lee Y-S. Hydrophilic modification of polyacrylonitrile membranes by oxyfluorination[J]. J Indust Eng Chem 2009; 15: 876-82. Available from: http://www.sciencedirect.com/science/ article/pii/S1226086X09001956 http://dx.doi.org/10.1016/j.jiec.2009.09.016

[11] Sukitpaneenit P, Chung T-S. Molecular design of the morphology and pore size of PVDF hollow fiber membranes for ethanol-water separation employing the modified poreflow concept[J]. J Membr Sc 2011; 374: 67-82. Available from: http://www.sciencedirect.com/science/article/pii/ S0376738811001839

http://dx.doi.org/10.1016/j.memsci.2011.03.016

[12] Saljoughi E, Amirilargani M, Mohammadi T. Effect of PEG additive and coagulation bath temperature on the morphology, permeability and thermal/chemical stability of asymmetric CA membranes[J]. Desalination 2010; 262: 7278. Available from: http://www.sciencedirect.com/science/ article/pii/S0011916410003619

http://dx.doi.org/10.1016/j.desal.2010.05.046

[13] Patsioura A, Galanakis CM, Gekas V. Ultrafiltration optimization for the recovery of $\beta$-glucan from oat mil waste[J]. J Membr Sci 2011; 373: 53-63. Available from: http://www.sciencedirect.com/science/article/pii/S037673881 1001529

http://dx.doi.org/10.1016/j.memsci.2011.02.032

[14] Conesa A, Gumi T, Palet C. Membrane thickness and preparation temperature as key parameters for controlling the macrovoid structure of chiral activated membranes (CAM) [J]. J Membr Sci 2007; 287: 29-40. Available from: http://www.sciencedirect.com/science/article/pii/S037673880 6006582

http://dx.doi.org/10.1016/j.memsci.2006.10.006

[15] Sun H, Qi D, Xu J, Zhe C. Fractionation of polysaccharides from rapeseed by ultrafiltration: Effect of molecular pore size and operation conditions on the membrane performance[J]. Separat Purificat Technol 2011; 80: 670-76. Available from: http://www.sciencedirect.com/science/article/pii/S138358661 1003881

http://dx.doi.org/10.1016/j.seppur.2011.06.038

[16] Prádầnos $P$, De Abajo $\mathrm{J}$, de la Campa JG, Hernández $A$. A comparative analysis of flux limit models for ultrafiltration membranes[J]. J Membr Sci 1995; 108: 129-42. Available from: $\quad$ http://www.sciencedirect.com/science/article/pii/ 0376738895001475

http://dx.doi.org/10.1016/0376-7388(95)00147-5

[17] Chew PG, Casey AJ, Johnsson SK. Protein quality and physico-functionality of Australian sweet lupin (Lupinus angustifolius cv. Gungurru) protein concentrates prepared by isoelectric precipitation or ultrafiltration[J]. Food Chem 2003; 83: 575-83. Available from: http://www.sciencedirect.com/ science/article/pii/S0308814603001560

http://dx.doi.org/10.1016/S0308-8146(03)00156-0 
[18] Zulkali MMD, Ahmad AL, Derek CJC. Membrane application in proteomic studies: Preliminary studies on the effect of $\mathrm{pH}$, ionic strength and pressure on protein fractionation[J]. Desalination 2005; 179: 381-90. Available from: http://www.sciencedirect.com/science/article/pii/S001191640 5003036

http://dx.doi.org/10.1016/j.desal.2004.11.084
[19] Kanani DM, Ghosh R. A constant flux based mathematical model for predicting permeate flux decline in constant pressure protein ultrafiltration[J]. J Membr Sci 2007; 290: 207-15. Available from: http://www.sciencedirect.com/ science/article/pii/S0376738806008593

http://dx.doi.org/10.1016/i.memsci.2006.12.030 